\title{
FETOPLACENTAL INSUFFICIENCY AS A REASON OF OFFSPRING OXIDATIVE STATUS DISTURBANCES*
}

\author{
N. Yu. Seliukova ${ }^{1,2}$, K. V. Misiura ${ }^{1}$, M. O. Boiko', S. P. Kustova ${ }^{1}$, \\ N. H. Grushanska ${ }^{3}$, P. V. Sharandak ${ }^{4}$, N. V. Medvedovska ${ }^{5}$ \\ ${ }^{1}$ SI «V. Danilevsky Institute for Endocrine Pathology Problems of NAMS of Ukraine», Kharkiv, Ukraine; \\ ${ }^{2}$ National University of Pharmacy, Kharkiv, Ukraine; \\ ${ }^{3}$ National University of Life and Environmental Sciences of Ukraine, Kiev, Ukraine; \\ ${ }^{4}$ Ministry for Development of Economy, Trade and Agriculture of Ukraine, Kiev, Ukraine; \\ ${ }^{5}$ Scientific and coordination department NAMS of Ukraine, Kiev, Ukraine \\ seliukova_nat@ukr.net
}

The main scientific purposes of physiologists and pathophysiologists investigations during many years are particularities of the individual organism developing and mechanisms of pathologies forming in mammalians including men. The list of diseases associated with the disturbances of the ontogenetic programs is constantly growing. Congenital disorders caused by teratogenic, embryotoxic, fetotoxic and stress factors during pregnancy represent the largest part of mentioned above diseases [1]. The integrative mechanisms controlled fetal developing, include mother, fetus and placenta, when different stress factors initiate compensatory adaptive response [2]. On the one hand, fetoplacental insufficiency (FPI) is widespread clinic syndrome caused by uncompen- sated morphological and functional placental changes, accompanies with the disturbances of uterus-placental blood circulation and, respectively, with trophic fails, distress and fetus hypoxia, that are manifested in fetus growing and developing modifications [3-5]. The reasons of FPI developing may be endogenous in relation to placenta (for example, disturbances of placenta forming due to hormonal fluctuations or extragenital pathologies) or extraneous (preeclampsia, cardio-vascular disease etc). On the other hand, the uterus blood vessels may be immature (in comparatively young mothers) or have secondary involution (in mature mothers). Independently of trigger factors, the therapy of FPI consists of vasoactive, tokolytic, metabolic (vitamins), antioxidants, membranostabilizers

* The research was carried out as part of investigation work at the SI «V. Danilevsky Institute for Endocrine Pathology Problems of National Academy of Medical Science of Ukraine» «Studying the effects of "passive smoking» mothers during pregnancy on endocrine-somatic phenotype of offspring (experimental research)» (State registration number: 0117U007187).

Institution, which financed the research: National Academy of Medical Science of Ukraine.

The authors assume responsibility for the published work.

The authors guarantee absence of competing interests and their own financial interest when carrying out the research and writing the article.

The manuscript was received by the editorial staff 2.12.2019. 
and medicines that influence on blood rheological properties [4].

Now, molecular and genetic mechanisms of feto-metabolic programming under influence of unfavorable fetal surrounding are actively investigated. The searching of new remedies for prophylaxis and treatment of these negative changes is carried out. The hypothesis of fetal programming was for the first time published in 1998 in D.J. Baker scientific works [6]. Later, the epigenetic programming of gametes, embryonic cells and tissues and stem cells developing during critical "window» (sensitive period) of ontogenesis was linked to phenomenon mentioned above. During this sensitive period, adverse impact on mother's organism causes disturbances of pregnancy, regulates fetal growing and leads to disease developing in later life [7, 8]. Epigenetic modifications, which represent an inheritance of gene expression without changes in sequence of DNA nucleotides, may be relatively stable during cells proliferation and, therefore, may persist over time. The base elements of epigenetic changes are patterns of DNA methylation, histones modifications (acetylation, methylation, phosphorilation, ubiquitylation etc.), genome imprinting, chromatin and non coding RNA remodeling.

According to the current ideas, the increasing of lipid peroxidation (LP) is important pathogenetic part of any stress influence, the cause of membranes structure and functioning disturbances including mitochondrial membranes. LP activates some stress-reacting signaling pathways, which is the base of future diseases development $[9,10]$. However, there are some controversial experimental data about fetal oxidative stress influence on forming sexdepending predisposition for developing some chronic pathologies with age, such as obesity, metabolic syndrome, endothelian dysfunction and cardio-vascular disease [11, 12]. Therefore, the aim of this scientific work was to determine the influence of the experimental fetoplacental insufficiency on the both sex offspring oxidative status during puberty and to estimate the efficiency of base and complex therapy during pregnancy.

\section{MATERIALS AND METHODS}

Experiment has been carried out in Vistar rats. Experimental animals have been kept in standard conditions of vivarium, under natural sources of light, standard feeding and water regime ad libitum. The investigation has been carried out according to the National «General Principles for Animal Research Ethics» (Ukraine, 2001) [13], which corresponds to the «European Convention for the Protection of Vertebrate Animals used for Experimental and Other Scientific Purposes» (Strasburg, 1985) [13].

The healthy, Vistar mature rat's females of young (3-4 months) and mature (8-10 months) reproductive age with normal four-to five days estrus cycles have been used for both sex offspring obtaining. The presence of the sperm cells in morning vaginal swabs has been considered to be the first day of pregnancy. 8 groups for 7 pregnant females in each have been formed:

1. Groups I and II - intact animals of young and mature reproductive age;

2. Group III and IV - females with experimental FPI of young and mature reproductive age accordingly;
3. Groups V and VI - young and mature animals with experimental FPI treated by pharmaceutical composition which contains nontoxic active pharmaceutical ingredients of FPI basic therapeutic group - amino acid (L-arginine), dicarbonic acid (succinic acid), vitamins (folic acid) and vasoactive drug (dipyridamole). Experimental animals have received treatment from 11 to 19 day of pregnancy.

4. Groups VII and VIII - young and mature animals with experimental FPI treated by drug of comparison - dipyridamole.

The modeling of FPI has been carried out by daily subcutaneous introduction of $50 \%$ tetrachlormethane oil solution in dose of $2 \mathrm{ml} / \mathrm{kg}$ of body weight from 12 to 18 day of pregnancy. Animals - offspring have been killed on the $50^{\text {th }}$ day of life (puberty period) by quick decapitation without general anesthesia to avoid negative effects on sex hormones level and antioxidant enzymes systems [14].

The primary LP products - conjugated diens [15] end secondary lipid peroxidation pro- 
duct - malondialdehyde [16] have been determined using spectrophotometric analysis. Catalase activity (EC 1.11.16) in liver homogenates has been evaluated using reaction with molybdenum salts [17]; Superoxide Dismutase (SOD) activity (SOD, (EC 1.15.1.11)) — using nitroblu tetrazolium test reaction [18]; Glutathione Peroxidase (GPx, (EC 1.11.1.69) has been determined by Glutathione Reductase reaction test [19] and quantities of recovered Glutathione has been evaluated by test with Ellman's reagent [20]. The determination of protein in samples has been carried out by Lowry C.H. et al. method in Miller G. L. modification.

Statistical analysis has been fulfilled by variable statistical methods using standard programs Biostat and Statistica (Stat Soft. Inc., USA). Determination of the characteristic's distribution in samples has been carried out using Shapiro-Wilk criterion; equality of dispersion's distribution of characteristics in groups - by Leven criterion. The comparison both groups with normal distribution has been carried out using standard parametric Student's t-criterion for independent samples. The nonparametric Mann-Whitney U test has been used for both groups when distributions are not equal; Newman-Keuls method — for multiple comparison procedure. Data given are arithmetic mean, standard error of the mean and the median. The divergence of characteristics has been considered to be statistically significant when $\mathrm{p} \leq 0.05$.

\section{RESULTS AND THEIR DISCUSSION}

Data obtained confirm, that FPI induced in second period of pregnancy, caused antioxidant enzymes disturbances in offspring-rats during puberty (table 1,2), which realized in serum primary and secondary lipid peroxidation products' changes (table 3).

It has been pointed out, that males - offspring born to mature mothers have demonstrated increased level of Catalase activity in puberty period, than males-offspring born to young mothers (see table 3). It has been supported by significant decreasing of testosterone levels (median was $3.90 \mathrm{nmol} / \mathrm{l}$ apart $23.80 \mathrm{nmol} / \mathrm{l}$, accordantly, $\mathrm{p}<0.05$, that may confirm substantial initial differences of placenta functioning under the conditions of different reproductive age with influence on the base enzymes of synthesis and/or metabolism

\section{Liver Catalase and Superoxide Dismutase activities in intact and experimental rats}

\begin{tabular}{|c|c|c|c|c|}
\hline \multirow{2}{*}{$\begin{array}{l}\text { Group, } \\
\mathbf{n}=7\end{array}$} & \multicolumn{2}{|c|}{$\begin{array}{l}\text { Catalase, } \\
\text { U/mg of protein }\end{array}$} & \multicolumn{2}{|c|}{$\begin{array}{c}\text { Superoxide Dismutase, } \\
\text { U/mg of protein }\end{array}$} \\
\hline & male & female & male & female \\
\hline $\mathrm{I}$ & $20.84 \pm 1.70$ & $33.71 \pm 4.33$ & $68.66 \pm 7.36$ & $64.59 \pm 5.93$ \\
\hline II & $\begin{array}{c}29.90 \pm 1.14 \\
\mathrm{P}_{\mathrm{I}-\mathrm{II}}<0.01\end{array}$ & $35.24 \pm 2.96$ & $71.97 \pm 5.27$ & $76.82 \pm 5.48$ \\
\hline III & $\begin{array}{c}28.04 \pm 1.85 \\
\mathrm{P}_{\text {I-III }}<0.05\end{array}$ & $29.85 \pm 3.65$ & $85.53 \pm 9.37$ & $61.98 \pm 6.68$ \\
\hline IV & $\begin{array}{l}22.56 \pm 2.57 \\
\mathrm{P}_{\text {II-IV }}<0.05\end{array}$ & $27.56 \pm 2.84$ & $64.26 \pm 6.96$ & $\begin{array}{l}58.76 \pm 4.86 \\
\mathrm{P}_{\text {II-IV }}<0.05\end{array}$ \\
\hline $\mathrm{V}$ & $24.98 \pm 1.12$ & $28.04 \pm 1.45$ & $\begin{array}{c}88.29 \pm 6.32 \\
0.05<\mathrm{P}_{\mathrm{I}-\mathrm{V}}<0.1\end{array}$ & $63.47 \pm 2.91$ \\
\hline VI & $26.49 \pm 2.76$ & $29.75 \pm 3.69$ & $\begin{array}{c}83.67 \pm 6.45 \\
0.05<\mathrm{P}_{\text {IV-VI }}<0.1\end{array}$ & $65.68 \pm 2.59$ \\
\hline VII & $23.67 \pm 1.55$ & $27.25 \pm 2.25$ & $84.57 \pm 7.45$ & $62.91 \pm 3.01$ \\
\hline VIII & $26.51 \pm 1.34$ & $\begin{array}{l}26.32 \pm 1.88 \\
\mathrm{P}_{\text {II-VIII }}<0.05\end{array}$ & $79.45 \pm 5.82$ & $\begin{array}{l}61.31 \pm 3.68 \\
\mathrm{P}_{\text {II-VIII }}<0.05\end{array}$ \\
\hline
\end{tabular}

Notes.

Statistically significant differences, $\mathrm{p} \leq 0.05$. 
of sex hormones [1, 3]. In addition, FPI caused Catalase activity increasing in males-offspring born to young mothers, while males-offspring born to mature mothers have demonstrated significant decreasing of the same activity. The same thing has been observed next to GPx activity decreasing and coincide with reduced Glutathione levels decreasing (see table 2).

Glutathione Peroxidase activity and recovered Glutathione liver level in intact and experimental offspring

\begin{tabular}{|c|c|c|c|c|}
\hline \multirow{2}{*}{$\begin{array}{l}\text { Group, } \\
\mathbf{n}=7\end{array}$} & \multicolumn{2}{|c|}{$\begin{array}{c}\text { Glutathione Peroxidase, } \\
\text { nmol/mg of protein }\end{array}$} & \multicolumn{2}{|c|}{$\begin{array}{c}\text { Recovered Glutathione, } \\
\mathrm{mkmol} / \mathrm{mg} \text { of protein }\end{array}$} \\
\hline & male & female & male & female \\
\hline I & $13.47 \pm 0.97$ & $9.83 \pm 0.50$ & $21.08 \pm 1.61$ & $21.02 \pm 2.12$ \\
\hline II & $14.08 \pm 1.07$ & $10.45 \pm 0.78$ & $18.59 \pm 0.82$ & $25.58 \pm 2.89$ \\
\hline III & $11.49 \pm 0.50$ & $10.86 \pm 0.50$ & $\begin{array}{c}12.90 \pm 1.37 \\
\mathrm{P}_{\mathrm{I}-\mathrm{III}}<0.01\end{array}$ & $\begin{array}{c}14.23 \pm 1.38 \\
\mathrm{P}_{\text {I-III }}<0.05\end{array}$ \\
\hline IV & $\begin{array}{l}10.47 \pm 0.70 \\
\mathrm{P}_{\text {II-IV }}<0.05\end{array}$ & $12.65 \pm 0.75$ & $\begin{array}{l}10.59 \pm 0.97 \\
\mathrm{P}_{\mathrm{II}-\mathrm{IV}}<0,001\end{array}$ & $\begin{array}{l}16.03 \pm 1.06 \\
\mathrm{P}_{\text {II-IV }}<0.05\end{array}$ \\
\hline $\mathrm{V}$ & $12.09 \pm 1.14$ & $\begin{array}{c}12.33 \pm 0.75 \\
\mathrm{P}_{\mathrm{I}-\mathrm{V}}<0.05\end{array}$ & $\begin{array}{c}16.57 \pm 0.63 \\
\mathrm{P}_{\text {I-V }}<0.05 \\
\mathrm{P}_{\text {III-V }}<0.05\end{array}$ & $18.30 \pm 1.17$ \\
\hline VI & $12.94 \pm 0.66$ & $11.94 \pm 1.15$ & $\begin{array}{l}15.35 \pm 0.89 \\
\mathrm{P}_{\text {II-VI }}<0.05 \\
\mathrm{P}_{\text {IV-VI }}<0.01\end{array}$ & $\begin{array}{l}17.72 \pm 0.59 \\
\mathrm{P}_{\text {II-VI }}<0.05\end{array}$ \\
\hline VII & $11.75 \pm 0.58$ & $11.81 \pm 0.57$ & $\begin{array}{l}14.86 \pm 0.74 \\
\mathrm{P}_{\mathrm{I}-\mathrm{VII}}<0.05\end{array}$ & $18.27 \pm 1.85$ \\
\hline VIII & $12.01 \pm 1.27$ & $\begin{array}{l}12.75 \pm 1.22 \\
\mathrm{P}_{\text {II-VIII }}<0.05\end{array}$ & $\begin{array}{l}15.58 \pm 1.33 \\
\mathrm{P}_{\text {IV-VIII }}<0.02\end{array}$ & $\begin{array}{l}16.81 \pm 0.33 \\
\mathrm{P}_{\text {II-VIII }}<0.02\end{array}$ \\
\hline
\end{tabular}

Notes.

Statistically significant differences, $\mathrm{p} \leq 0.05$.

\section{Serum Lipid Peroxidation products in intact and experimental offspring}

\begin{tabular}{|c|c|c|c|c|}
\hline \multirow{2}{*}{$\begin{array}{c}\text { Group, } \\
\mathbf{n}=7\end{array}$} & \multicolumn{2}{|c|}{ Conjugated diens, $\mathrm{mmol} / \mathrm{l}$} & \multicolumn{2}{|c|}{ Malondialdehyde, mmol/l } \\
\hline & male & female & male & female \\
\hline I & $0.175 \pm 0.012$ & $0.145 \pm 0.013$ & $0.96 \pm 0.09$ & $0.79 \pm 0.06$ \\
\hline II & $0.195 \pm 0.009$ & $0.118 \pm 0.006$ & $1.08 \pm 0.04$ & $0.73 \pm 0.06$ \\
\hline III & $\begin{array}{c}0.259 \pm 0.021 \\
\mathrm{P}_{\text {I-III }}<0.01\end{array}$ & $\begin{array}{c}0.228 \pm 0.014 \\
\mathrm{P}_{\text {I-III }}<0.01\end{array}$ & $\begin{array}{l}1.45 \pm 0.11 \\
\mathrm{P}_{\text {I-III }}<0.01\end{array}$ & $\begin{array}{l}1.13 \pm 0.05 \\
\mathrm{P}_{\text {I-III }}<0.01\end{array}$ \\
\hline IV & $0.218 \pm 0.014$ & $\begin{array}{c}0.198 \pm 0.011 \\
\mathrm{P}_{\text {II-IV }}<0.001\end{array}$ & $\begin{array}{l}1.58 \pm 0.14 \\
\mathrm{P}_{\text {II-IV }}<0.01\end{array}$ & $\begin{array}{c}1.17 \pm 0.07 \\
\mathrm{P}_{\text {II-IV }}<0.002\end{array}$ \\
\hline $\mathrm{V}$ & $\begin{array}{c}0.197 \pm 0013 \\
\mathrm{P}_{\text {III-V }}<0.05\end{array}$ & $\begin{array}{c}0.173 \pm 0.015 \\
\mathrm{P}_{\text {III-V }}<0.05\end{array}$ & $\begin{array}{l}1.05 \pm 0.07 \\
\mathrm{P}_{\text {III-V }}<0.02\end{array}$ & $\begin{array}{c}0.99 \pm 0.05 \\
\mathrm{P}_{\mathrm{I}-\mathrm{V}}<0.05\end{array}$ \\
\hline VI & $0.204 \pm 0.012$ & $\begin{array}{c}0.204 \pm 0.009 \\
\mathrm{P}_{\text {II-VI }}<0.001\end{array}$ & $\begin{array}{l}1.14 \pm 0.05 \\
\mathrm{P}_{\text {IV-VI }}<0.02\end{array}$ & $\begin{array}{l}1.05 \pm 0.04 \\
\mathrm{P}_{\text {II-VI }}<0.01\end{array}$ \\
\hline VII & $\begin{array}{c}0.226 \pm 0.016 \\
\mathrm{P}_{\mathrm{I}-\mathrm{VII}}<0.05\end{array}$ & $\begin{array}{c}0.199 \pm 0.016 \\
\mathrm{P}_{\text {I-VII }}<0.05\end{array}$ & $1.17 \pm 0.08$ & $\begin{array}{l}1.10 \pm 0.08 \\
\mathrm{P}_{\text {I-VII }}<0.02\end{array}$ \\
\hline VIII & $0.206 \pm 0.014$ & $\begin{array}{l}0.206 \pm 0.011 \\
\mathrm{P}_{\text {II-VIII }}<0.001\end{array}$ & $1.25 \pm 0.10$ & $\begin{array}{c}1.11 \pm 0.06 \\
\mathrm{P}_{\text {II-VIII }}<0.01\end{array}$ \\
\hline
\end{tabular}

Notes.

Statistically significant differences, $\mathrm{p} \leq 0.05$. 
It should be noted, that Glutathione content has decreased in both offspring groups under the influence of FPI. According to the recent trends, compensatory enzymes activation in offspring undergone different stress factors during pregnancy is linked to epigenetic activating of stress-reacting pathways that further may lead to the resources exhaustion and chronic inflammatory disease development $[7,8]$. On the other hand, the inhibition protecting systems components or different metabolic enzymes are often linked to protein molecules modifying during ontogenesis [9, 10]. Liver Catalase activity in females-offspring was relatively constant, while SOD activity decreasing has been detected in offspring born to mature mothers with FPI (see table 1).

Combined therapy has mostly normalized antioxidant system's indices, but not complete- ly, that has been confirmed by serum LP products levels which have not reached values of intact animals (see table 3).

Therefore, it should be concluded, that FPI cause significant changes of base elements of the antioxidant defense in both sex offspring, but mostly in males-offspring.

Taking into consideration, that using vasodilator medicine separately, as well as in combination with additional components, has reliably recovered evaluated indices, these metabolic disorders may be primary linked to chronic fetus hypoxia.

Besides, the most significant changes have been observed in both sex offspring born to mature mothers that may be explained by influence of placental involution which causes trophic disorders.

\section{CONCLUSIONS}

1. FPI in second period of pregnancy cause changes of antioxidant enzymes system's activity in both sex rats-offspring, but mostly in females-offspring during puberty. Antioxidant enzymes system's changes manifest as increasing levels of primary and secondary lipid peroxidation products.
2. The most significant disturbances have been observed in both sex offspring born to mature mothers that may be explained by placental involution.

3. Combined therapy has normalized offspring's oxidative status more effective than vasodilator as monotherapy.

\section{REFERENCES}

1. Reznikov AG, Pishak VP, Nosenko ND, et al. Prenatal'nyj stress i nejrojendokrinnaja patologija, Chernovcy, 2004: $318 \mathrm{p}$

2. Petraglia F, Imperatore A, Challis JRG. Endocrine Rev 2010; 31(6): 783-816. doi:10.1210/er.2009-0019

3. Chepka JL. Ukr Med Chasopys 2001; 6: 108-115.

4. Lihachov VK, Petrenko JV. Problemy, dostizhenija i perspektivy razvitija mediko-biologicheskih nauk i prakticheskogo zdravoohranenija: Tr. KGMU.ym. S. Y. Georgyeuskogo, Symferopol 2009; 145(3): 151-155.

5. Shherbakov AJ, Tihaja IA, Shherbakov VJ, Novikova EA. Mezhdunar Med Zhurn 2012; 3: 50-53.

6. Baker DJ. Clin Sci 1998; 95: 115-128.

7. Cheedipudi S, Genolet O, Dobreva G. Front Genet 2014; 5: 19. doi: 10.3389/ fgene.2014.00019

8. Zhu Z, Cao F, Li X. Front Endocrinol 2019; 10: 764. doi: 10.3389/fendo.2019.00764

9. Sahin E, DePinho RA. Nat Rev Mol Cell Biol 2012; 13(6): 397-404. doi: 10.1038/nrm3352

10. Menezo YJ, Silvestris E, Dale B, Elder K. Reprod Biomed Online 2016; 33(6): 668-683. doi: 10.1016/j.rbmo. 2016.09.006
11. Dimova LG, Battista S, Plosch T, et al. Redox Biol 2020; 28: 101329. doi: 10.1016/j.redox.2019.101329

12. Badran M, Yassin BA, Lin DTS, et al. J Physiol 2019; 597(22): 5349-5364. doi: 10.1113/JP277936

13. Reznikov OG. Endokrynologija 2003; 8(1): 142-145.

14. Jakovljeva LV, Zajchenko GV, Cypkun AG, et al. Doklinichne vyvchennja likars'kyh zasobiv, pryznachenyh dlja likuvannja placentarnoi' dysfunkcii': metod. Rekomendacii', Kyi'v, 2009.

15. Placer Z, Vidlakova M, Kupila L. Chehosl Med Obzor 1970; 16(1): 30-34.

16. Stal'naja ID, Garishvili GT. Sovremennye metody biohimii, Moskva, 1977: 43-44.

17. Koroljuk A, Ivanova L, Majorova I, Tokarev VE. Lab Delo 1988; 1: 16-17.

18. Chevari S, Andjal T, Shtrenger J. Lab Delo 1991; 10: 9-13.

19. Lankin VZ, Tihadze AN, Kovalevskaja AL, et al. Doklady AN SSSR 1981; 261(3): 18-24.

20. Beutler E, Duron O, Kelly B. Lab Clin Med 1963; 63(5): 882-888. 


\section{ФЕТОПЛАЦЕНТАРНА НЕДОСТАТНІСТЬ ЯК ПРИЧИНА ПОРУШЕНЬ ОКСИДАТИВНОГО СТАТУСУ У НАЩАДКІВ}

Селюкова Н. Ю. ${ }^{1,2}$, Місюра К. В. ${ }^{1}$, Бойко М. О. ${ }^{1}$,

Кустова С. П. ${ }^{1}$, Грушанська Н. Г. ${ }^{3}$, Шарандак П. В. ${ }^{4}$, Медведовська Н. В. ${ }^{5}$

${ }^{1}$ ДУ «Інститут проблем ендокринної патологї ім. В. Я. Данилевського НАМН Украйни», м. Харків, Україна;

${ }^{2}$ Національний фбармацевтичний університет, м. Харків, Украӥна;

${ }^{3}$ Національний університет біоресурсів і природокористування України, м. Київ, Украӥна;

${ }^{4}$ Міністерство розвитку еконоліки, торгівлі та сільського господарства Украӥни, м. Київ, Україна;

${ }^{5}$ Науково-координаційне управління апарату президії НАМН України, м. Київ, Україна seliukova_nat@ukr.net

Визначили вплив експериментальної фетоплацентарної недостатності у щурів молодого та зрілого репродуктивного віку на формування оксидативного статусу нащадків обох статей в період пубертату та оцінили ефекти базової та комплексної медикаментозної терапії під час вагітності. Виявлено, що фетоплацентарна недостатність у другій половині вагітності призводить до формування у нащадків обох статей, але більшою мірою у нащадків-самців, в період пубертату зміненого патерна активності ферментів антиоксидантної системи, що реалізуеться у зростанні рівнів як первинних, так і кінцевих продуктів ліпопероксидації і може бути підгрунтям для розвитку у подальшому хронічних захворювань. Більш виразні порушення спостерігалися у нащадків матерів зрілого репродуктивного віку, що може бути обумовлено додатковим впливом інволютивних процесів у плаценті. Застосування судинорозширювального препарату окремо та, більш ефективно, у комбінації, статистично значуще відновлювало досліджені показники.

К лючов і слова: фетоплацентарна недостатність, нащадки, оксидативний статус, терапія.

\section{ФЕТОПЛАЦЕНТАРНАЯ НЕДОСТАТОЧНОСТЬ КАК ПРИЧИНА НАРУШЕНИЙ ОКСИДАТИВНОГО СТАТУСА У ПОТОМКОВ}

Селюкова Н. Ю. ${ }^{1,2}$, Мисюра К. В. ${ }^{1}$, Бойко М. А. ${ }^{1}$,

Кустова С. П. ${ }^{1}$, Грушанская Н. Г. ${ }^{3}$, Шарандак П. В. ${ }^{4}$, Медведовская Н. В. ${ }^{5}$

${ }^{1}$ ГУ «Институт проблел эндокринной патологии им. В. Я. Данилевского НАМН Украины»,, г. Харьков, Украина;

${ }^{2}$ Национальний фбарлацевтический университет, г. Харьков, Украина;

${ }^{3}$ Национальный университет биоресурсов и природопользования Украины, г. Киев, Украина;

${ }^{4}$ Министерство развития экономики, торговли и сельского хозяйства Украины, г. Киев, Украина;

${ }^{5}$ Научно-координационное управление апарата президиума НАМН Украинь, г. Киев, Украина seliukova_nat@ukr.net

Определили влияние экспериментальной фетоплацентарной недостаточности у крыс молодого и зрелого репродуктивного возраста на формирование оксидативного статуса потомков обоего пола в период пубертата и оценили әффекты базовой и комплексной медикаментозной терапии во время беременности. Выявлено, что фетоплацентарная недостаточность во второй половине беременности приводит к формированию у потомков обоего пола, но в большей степени у потомков-самцов, в период пубертата измененного паттерна активности ферментов антиоксидантной системы, что реализуется в росте уровней как первичных, так и конечных продуктов липопероксидации и может быть основой для развития в дальнейшем хронических заболеваний. Более выраженные нарушения наблюдались у потомков матерей зрелого репродуктивного возраста, что может быть обусловлено дополнительным влиянием инволютивных процессов в плаценте. Применение сосудорасширяющего препарата в отдельности и, более эффективно в комбинации, статистически значимо восстанавливало изученные показатели.

Ключевые слова: фетоплацентарная недостаточность, потомки, оксидативный статус, терапия. 


\title{
FETOPLACENTAL INSUFFICIENCY AS A REASON OF OFFSPRING OXIDATIVE STATUS DISTURBANCES
}

\author{
N. Yu. Seliukova ${ }^{1,2}$, K. V. Misiura ${ }^{1}$, M. O. Boiko ${ }^{1}$, \\ S. P. Kustova ${ }^{1}$, N. H. Grushanska ${ }^{3}$, P. V. Sharandak ${ }^{4}$, N. V. Medvedovska ${ }^{5}$ \\ ${ }^{1}$ SI «V. Danilevsky Institute for Endocrine Pathology Problems of NAMS of Ukraine», \\ Kharkiv, Ukraine; \\ ${ }^{2}$ National University of Pharmacy, Kharkiv, Ukraine; \\ ${ }^{3}$ National University of Life and Environmental Sciences of Ukraine, Kiev, Ukraine; \\ ${ }^{4}$ Ministry for Development of Economy, Trade and Agriculture of Ukraine, Kiev, Ukraine; \\ ${ }^{5}$ Scientific and coordination department NAMS of Ukraine, Kiev, Ukraine \\ seliukova_nat@ukr.net
}

The aim of this scientific work was to determine the influence of the experimental fetoplacental insufficiency on the both sex offspring oxidative status during puberty and to estimate the efficiency of base and complex therapy during pregnancy.

Materials. The healthy, Vistar mature rat's females of young (3-4 months) and mature (8-10 months) reproductive age have been used for both sex offspring obtaining. 8 groups for 7 pregnant females in each have been formed: Groups I and II - intact animals of young and mature reproductive age; Group III and IV - females with experimental Fetoplacental insufficiency (FPI) of young and mature reproductive age accordingly; Groups V and VI - young and mature animals with experimental FPI treated by pharmaceutical composition which contains nontoxic active pharmaceutical ingredients of FPI basic therapeutic group - amino acid (L-arginine), dicarbonic acid (succinic acid), vitamins (folic acid) and vasoactive drug (dipyridamole). Experimental animals have received treatment from 11 to 19 day of pregnancy. Groups VII and VIII - young and mature animals with experimental FPI treated by drug of comparison - dipyridamole. The modeling of FPI has been carried out by daily subcutaneous introduction of $50 \%$ tetrachlormethane oil solution in dose of $2 \mathrm{ml} / \mathrm{kg}$ of body weight from 12 to 18 day of pregnancy. Animals - offspring have been killed on the $50^{\text {th }}$ day of life (puberty period) by quick decapitation without general anesthesia to avoid negative effects on sex hormones level and antioxidant enzymes systems.

Results. The effect of experimental fetoplacental insufficiency in rats of young and mature reproductive age on the oxidative status formation in offspring of both sexes during puberty was determined and the effects of basic and complex therapy during pregnancy were evaluated. It was revealed that fetoplacental insufficiency in the second half of pregnancy leads to the formation in offspring of both sexes, but more pronounced in male offspring, during the puberty, an altered pattern of the antioxidant system enzymatic activity, which is realized in increased levels of both primary and final products of lipoperoxidation and may be the basis for further development of chronic diseases. More pronounced disorders were observed in the offspring of mothers of mature reproductive age, which may be due to the additional influence of involutive processes in the placenta. The use of a vasodilator drug alone and, more effectively in combination, significantly restored the studied parameters.

Keyw ords: fetoplacental insufficiency, offspring, oxidative status, therapy. 